\begin{tabular}{|l|l|l|c|c|}
\hline Clinical Study & $\begin{array}{l}\text { R E van Genugten and } \\
\text { others }\end{array}$ & $\begin{array}{l}\text { Sitagliptin to treat GC islet } \\
\text { dysfunction }\end{array}$ & $\mathbf{1 7 0 : 3}$ & $\mathbf{4 2 9 - 4 3 9}$
\end{tabular}

\title{
Does dipeptidyl peptidase-4 inhibition prevent the diabetogenic effects of glucocorticoids in men with the metabolic syndrome? A randomized controlled trial
}

\author{
Renate E van Genugten ${ }^{1}$, Daniël H van Raalte', Marcel H Muskiet ${ }^{1}$, \\ Martijn W Heymans ${ }^{2}$, Petra J W Pouwels ${ }^{3}$, D Margriet Ouwens ${ }^{4,5}$, \\ Andrea Mari' ${ }^{6}$ and Michaela Diamant ${ }^{1}$
}

${ }^{1}$ Department of Internal Medicine, Diabetes Center, ${ }^{2}$ Department of Methodology and Applied Biostatistics, Institute of Health Sciences and ${ }^{3}$ Department of Physics and Medical Technology, VU University Medical Center, PO Box 7057, 1007 MB Amsterdam, The Netherlands, ${ }^{4}$ Institute of Clinical Biochemistry and Pathobiochemistry, German Diabetes Center, Düsseldorf, Germany, ${ }^{5}$ Department of Endocrinology, Ghent University Hospital, Ghent, Belgium and ${ }^{6}$ Institute of Biomedical Engineering, National Research Council, Padova, Italy
Correspondence should be addressed to D H van Raalte

Email

d.vanraalte@vumc.nl

\begin{abstract}
Objective: Anti-inflammatory glucocorticoid (GC) therapy often induces hyperglycemia due to insulin resistance and islet-cell dysfunction. Incretin-based therapies may preserve glucose tolerance and pancreatic islet-cell function. In this study, we hypothesized that concomitant administration of the dipeptidyl peptidase-4 inhibitor sitagliptin and prednisolone in men at high risk to develop type 2 diabetes could protect against the GC-induced diabetogenic effects.

Design and methods: Men with the metabolic syndrome but without diabetes received prednisolone 30 mg once daily plus sitagliptin $100 \mathrm{mg}$ once daily $(n=14)$, prednisolone $(n=12)$ or sitagliptin alone $(n=14)$ or placebo $(n=12)$ for 14 days in a double-blind $2 \times 2$ randomized-controlled study. Glucose, insulin, C-peptide, and glucagon were measured in the fasted state and following a standardized mixed-meal test. $\beta$-cell function parameters were assessed both from a hyperglycemic-arginine clamp procedure and from the meal test. Insulin sensitivity ( $M$-value) was measured by euglycemic clamp.

Results: Prednisolone increased postprandial area under the curve (AUC)-glucose by $17 \%(P<0.001$ vs placebo) and postprandial AUC-glucagon by $50 \%(P<0.001)$. Prednisolone reduced 1 st and 2 nd phase glucose-stimulated- and combined hyperglycemia-arginine-stimulated $C$-peptide secretion (all $P \leq 0.001$ ). When sitagliptin was added, both clamp-measured $\beta$-cell function ( $P=$ NS for 1 st and 2 nd phase vs placebo) and postprandial hyperglucagonemia $(P=$ NS vs placebo) remained unaffected. However, administration of sitagliptin could not prevent prednisolone-induced increment in postprandial glucose concentrations $(P<0.001$ vs placebo). $M$-value was not altered by any treatment.

Conclusion: Fourteen-day treatment with high-dose prednisolone impaired postprandial glucose metabolism in subjects with the metabolic syndrome. Concomitant treatment with sitagliptin improved various aspects of pancreatic islet-cell function, but did not prevent deterioration of glucose tolerance by GC treatment.
\end{abstract}

\section{Introduction}

Glucocorticoids (GCs), such as prednisolone, are potent anti-inflammatory agents frequently used to treat inflammatory conditions such as rheumatoid arthritis, inflammatory bowel disease, or chronic obstructive pulmonary disease, together accounting for $\sim 10$ million oral prescriptions per year in the USA (1). However, GCs (c) 2014 European Society of Endocrinology Printed in Great Britain
Published by Bioscientifica Ltd. 
are diabetogenic drugs as their use is associated with the development of hyperglycemia and overt diabetes with a prevalence up to $30 \%(2,3)$. Given the large number of prescribed GC drugs, this represents a significant disease burden. The diabetogenic effects have classically been attributed to the induction of peripheral insulin resistance (4). Recent studies have indicated that GCs additionally impair $\alpha$ and $\beta$-cell function $(5,6,7)$. In particular, postprandial metabolism is disturbed by GC treatment, resulting in characteristic hyperglycemia during the day and evening $(8,9)$.

In contrast to other GC-induced side effects such as osteoporosis (10) or gastric ulcer disease (11), for which protective agents are routinely prescribed at the initiation of GC therapy, no such strategies are currently used to prevent hyperglycemia and protect islet-cell function. Given the more recent insights that GC therapy particularly affects postprandial glucose metabolism by impairing islet-cell function, we hypothesized that incretin-based therapies could be effective to prevent these side effects. Indeed, both glucagon-like peptide 1 (GLP1) receptor agonists and dipeptidyl peptidase-4 (DPP-4) inhibitors mainly target postprandial hyperglycemia by stimulating insulin- and suppressing glucagon secretion in a glucosedependent manner (12). Moreover, compounds from both classes have shown to improve various aspects of pancreatic islet-cell function in humans with type 2 diabetes $(13,14)$. In a proof-of-principle study, i.v. administration of the GLP1 receptor agonist, exenatide, prevented postprandial hyperglycemia and islet-cell dysfunction induced by high-dose of prednisolone treatment for 2 days in healthy young men (15). In a clinical setting, however, high-dose GCs are often prescribed for a prolonged period of time, before tapering down, to middle-aged patients suffering from low-to-high grade inflammation (16). In addition, in daily practice, the use of GLP1 receptor agonists for the prevention of steroiddiabetes may be less acceptable for patients because these drugs are injected and are associated with a high rate of gastrointestinal side effects. In contrast, oral DPP-4 inhibitors are well tolerated (17). Finally, incretin-based therapies have been shown to have beneficial or neutral effects on several cardiovascular risk factors (18). This is of additional interest as GC treatment is linked to increased cardiovascular risk through, amongst others, inducing hypertension, obesity, or dyslipidemia (19).

In this study, we hypothesized that concomitant administration of the DPP-4 inhibitor sitagliptin and high-dose prednisolone in men at high risk to develop type 2 diabetes would protect against the GC-induced diabetogenic effects, by preserving glucose tolerance and islet-cell function.

\section{Research design and methods}

\section{Study design}

The study was a randomized, placebo-controlled, $2 \times 2$ factorial, double-blind clinical trial (NCT00721552). After a 4-week run-in period, participants were randomized to: i) sitagliptin $100 \mathrm{mg}$ once daily plus prednisolone $30 \mathrm{mg}$ once daily (PRED +SITA); ii) prednisolone $30 \mathrm{mg}$ once daily plus sitagliptin-placebo (PRED); iii) sitagliptin $100 \mathrm{mg}$ once daily plus prednisolone-placebo (SITA); or iv) prednisolone-placebo plus sitagliptin-placebo (PBO).

Participants were treated for 14 days with sitagliptin (-placebo) plus prednisolone(-placebo). From days 15 to 28 , the prednisolone-arm was discontinued (i.e. to ascertain the reversibility of the PRED-induced cardiometabolic abnormalities), while participants continued the intake of sitagliptin(-placebo) in order to study the effects of sitagliptin on restoration of GC-induced glucose intolerance and cardiovascular risk. After screening for eligibility and inclusion, all participants underwent the study procedures (see below) both before randomization and during treatment (days 13 and 14; Supplementary Fig. 1A, see section on supplementary data given at the end of this article). At days 7 and 28 of treatment, safety visits were performed and study medication was counted. The study was performed according to the Declaration of Helsinki and approved by the Local Ethics Review Board. Furthermore, all subjects provided written informed consent before participation.

\section{Participants}

Participants with the metabolic syndrome were recruited by advertisement in local newspapers. The metabolic syndrome was defined according to International Diabetes Federation (IDF) criteria (20), except that individuals were not allowed to have type 2 diabetes (defined as fasting plasma glucose (FPG) $>7.0 \mathrm{mmol} / \mathrm{l}$ and 2-h plasma glucose $>11.0 \mathrm{mmol} / \mathrm{l})$. No other comorbidities, including a history of cardiovascular disease or treatment with agents other than anti-hypertensive drugs and statins were allowed.

\section{Screening visit and anthropometric measurements}

Eligibility was assessed by medical history, anthropometric measurements, physical examination, standard laboratory assessments in the fasting state, and a 2-h 75-g 
oral glucose tolerance test (OGTT). The participants were instructed to omit their usual medication on the morning of study visits and to refrain from eating, drinking (other than water), and smoking after $2200 \mathrm{~h}$ the evening before the scheduled visit.

\section{Mixed-meal test}

The standardized mixed-meal test procedure was performed before randomization at baseline (day 2) and at day 14 of medication intake. The individuals were instructed to arrive at the research unit in the morning at $0800 \mathrm{~h}$ after a 10-h overnight fast. In addition, subjects had not performed strenuous exercise $48 \mathrm{~h}$ before the study visit. A cannula was inserted into the antecubital vein of the dominant arm to allow blood sampling. After completion of the fasting blood collections ( $T=-15 \mathrm{~min}$ ), the standardized mixed-meal, consisting of $905 \mathrm{kcal}$ (75 g carbohydrates, $36 \mathrm{~g}$ proteins, and $50 \mathrm{~g}$ fat) (15), was served. The participants were instructed to consume the meal within $10 \mathrm{~min}$. At prespecified time points blood samples were obtained to assess glucose, insulin, C-peptide, and glucagon (Supplementary Fig. 1B). Whole-blood glucose was assessed directly (see below), other blood samples were collected, processed, and stored at $-80{ }^{\circ} \mathrm{C}$ until analysis. Furthermore, in the fasting state, DPP-4 activity was determined.

\section{Combined euglycemic-hyperglycemic clamp procedure}

Before randomization (day 1) and at day 13 of treatment, a combined hyperinsulinemic-euglycemic and hyperglycemic-plus-arginine clamp procedure was performed to assess peripheral insulin sensitivity and $\beta$-cell function (21). After an overnight fast, insulin infusion at a rate of $40 \mathrm{mU} / \mathrm{m}^{2}$ per min was started for $120 \mathrm{~min}$ with plasma glucose levels kept at $5 \mathrm{mM}$. One hour after discontinuation of insulin infusion, plasma glucose was raised to $15 \mathrm{mM}$ ( $T=180 \mathrm{~min}$ ) by a glucose bolus and was kept at this level for the remainder of the test. Following $80 \mathrm{~min}$ of hyperglycemia $(T=260 \mathrm{~min})$, an i.v. bolus of $5 \mathrm{~g}$ arginine was given to assess maximum secretory capacity at this level of glycemia.

\section{Cardiovascular risk factors}

At baseline (day 2), day 14, and day 28 body weight, BMI, body fat percentage, blood pressure, and fasting lipid profiles (total cholesterol, LDL-cholesterol, HDL-cholesterol, and triglycerides) were measured. Blood pressure was assessed as the mean of three measurements (within $30 \mathrm{~min}$, at least $5 \mathrm{~min}$ apart), measured manually by the same investigator, in a seated position at the nondominant arm, using a calibrated sphygmomanometer (Pressostabil, Welch Allyn, Bucks, UK). Body fat percentage was assessed by a four-electrode bio-impedance analyzer (BF-906, Maltron International, Essex, UK). At days 2 and 28 , the effect of SITA treatment on body fat distribution was assessed by manual measurement of waist circumference $(\mathrm{cm})$ and of subcutaneous and visceral adipose tissue area $\left(\mathrm{cm}^{2}\right)$ by magnetic resonance imaging at the level of L4-L5 (as described previously (22)). In addition, ${ }^{1} \mathrm{H}$-spectroscopy was performed to assess hepatic and pancreatic triglyceride content (as described previously (22)). Furthermore, at day 28 the 2-h 75-g OGTT was repeated to assess whether all participants returned to baseline glucose tolerance, and whether this was different between PRED+SITA and PRED.

\section{Biochemical measurements}

Whole-blood glucose concentrations during the meal and clamp tests were assessed immediately after collection by glucose oxidase method using YSI2300 STAT Plus analyzer (YSI, Yellow Springs, OH, USA). Plasma levels of insulin and C-peptide were determined using an immunometric assay (Luminescence, Advia Centaur, Siemens Medical Solution Diagnostics, Malvern, PA, USA) and plasma glucagon by using a RIA (Linco Research, St Louis, MO, USA). Plasma DPP-4 activity was measured by a luminescent assay using Gly-Pro-aminoluciferin as substrate (DPPIV-Glo Protease Assay, Promega). Other laboratory values (plasma glucose (during OGTT), HbA1c, and lipid profiles) were analyzed by the Department of Clinical Chemistry of the VU University Medical Centre.

\section{Calculations}

Glucose tolerance - As a measure of glucose tolerance, 4-h postprandial area under the curve (AUC) for glucose was calculated by the trapezoidal method.

Meal-derived islet-cell function parameters $\boldsymbol{~ T o}$ assess $\beta$-cell function, we calculated $4 \mathrm{~h}$-AUCs for insulin and C-peptide, and $4 \mathrm{~h}-\mathrm{AUC}_{\text {insulin:glucose ratio. To assess }}$ $\alpha$-cell function, we calculated $4 \mathrm{~h}$-AUC $\mathrm{Clucagon}_{\text {and }}$

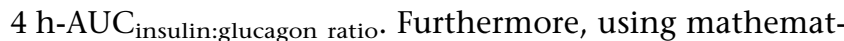
ical modeling $(23,24)$ the relationship between insulin release and (plasma) glucose concentration was described as the sum of two components, generating a total of four 
measures of $\beta$-cell function. The first component expresses the static relationship between insulin secretion and glucose concentrations. From this dose-response function the insulin secretion rate at a fixed glucose reference (of $5.5 \mathrm{mM}$ ) can be calculated as well as the slope of this relationship, which represents the overall $\beta$-cell glucose sensitivity. However, this relationship between insulin secretion and prevailing glucose concentration is modulated by a time-varying factor, which is quantified as the potentiation factor ratio. The potentiation factor encompasses all factors that may modulate insulin secretion (glucose and nonglucose substrates, gastrointestinal hormones, and neuromodulation). The second component of the model is the dynamic change in insulin secretion that depends on the rate of change in glucose concentrations, which is called the rate sensitivity.

Clamp-derived $\beta$-cell function variables $~$ Hyperglycemic clamp-derived $\beta$-cell function was assessed as 1 st and 2nd phase glucose- and combined hyperglycemiaarginine-stimulated C-peptide secretion calculated as

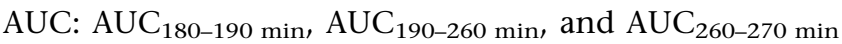
respectively (nmol/min per $\mathrm{l}$ ).

Insulin sensitivity $\bullet$ From the final $30 \mathrm{~min}$ of the euglycemic-clamp procedure, we calculated insulin sensitivity as the $M$-value ( $\mathrm{mg} / \mathrm{kg}$ per min) (21). In addition, in order to be able to compare our results with previous studies, an approximation of whole-body insulin sensitivity was assessed in the fasting state by means of the homeostasis model assessment of insulin resistance (HOMA2-IR) (25).

\section{Statistical analysis and sample size calculation}

Owing to small numbers and skewed distribution, data are presented as median values with interquartile range at baseline (day 2 or 1 ) and post-treatment (day 13, 14, or 28 ). For each parameter, we calculated the ratio to pretreatment, i.e. the relative change from baseline (post-/pre-treatment value). Owing to small group sizes and unequal distribution among groups, group-differences were nonparametrically tested by Kruskal-Wallis; in case of significance subsequent Mann-Whitney $U$ for betweengroup analysis with post-hoc Bonferroni's correction was performed (comparisons of interest: PRED vs PBO, PRED+ SITA vs PBO, and SITA vs PBO). Statistical analyses were performed using SPSS, version 20.0 for Windows, and $P<0.05$ was considered statistically significant.

Justification of sample size $\bullet$ For between-group differences in the primary objective, i.e. change in postprandial $\mathrm{AUC}_{\text {glucose, }}$ we expected a $30 \%$ change for treatment with prednisolone (based on our previous study (5)) which rendered a sample size of 8 to be sufficient to detect a 30\% change (s.D. 20\%) with a two-sided $\alpha$-level of 0.05 and a power of $80 \%$. For the effect of sitagliptin, we based our sample size calculation on previous studies in which DPP-4 inhibition was administered to subjects with impaired glucose metabolism $(26,27)$. We expected a $30 \%$ reduction in $\mathrm{AUC}_{\text {glucose }}$ (S.D. $25 \%$ ), therefore, with a two-sided $\alpha$-level of 0.05 and a power of $80 \%, 15$ subjects would be enough to detect the expected difference.

\section{Results}

\section{Subjects}

Of the 91 men screened (from February 2009 until April 2012), 54 fulfilled all eligibility criteria. The participants were randomized to one of the four medication groups (Supplementary Fig. 2, see section on supplementary data given at the end of this article). Table 1 provides the baseline characteristics of all participants. All participants were white and of male sex. No differences were observed among the groups at baseline (data not shown). Owing to inclusion criteria, participants met either three, four or five criteria for the metabolic syndrome. In total,

Table 1 Baseline characteristics of the study participants during the screening visit. Values are displayed as median (interquartile range) or proportion (\%).

\section{Characteristics}

Age (years)

Weight $(\mathrm{kg})$

BMI $\left(\mathrm{kg} / \mathrm{m}^{2}\right)$

Waist circumference $(\mathrm{cm})$

Fasting plasma glucose $(\mathrm{mmol} / \mathrm{l})$

2-h Plasma glucose $(\mathrm{mmol} / \mathrm{l})^{\mathrm{a}}$

HbA1c (\%)

$\mathrm{HbA} 1 \mathrm{c}(\mathrm{mmol} / \mathrm{mol})$

Total cholesterol $(\mathrm{mmol} / \mathrm{l})$

LDL-cholesterol ( $\mathrm{mmol} / \mathrm{l})$

HDL-cholesterol ( $\mathrm{mmol} / \mathrm{l})$

Triglycerides $(\mathrm{mmol} / \mathrm{l})$

Systolic blood pressure $(\mathrm{mmHg})$

Diastolic blood pressure $(\mathrm{mmHg})$

Use of anti-hypertensive drugs (\%)

Use of lipid-lowering drugs (\%)

Current smoker (\%)

\begin{tabular}{c}
\hline Values \\
\hline $58(51,62)$ \\
$103.1(95.1,108.9)$ \\
$31.2(29.4,33.7)$ \\
$112.5(106.0,118.5)$ \\
$5.9(5.5,6.1)$ \\
$6.3(5.2,8.0)$ \\
$5.6(5.4,5.7)$ \\
$38(36,39)$ \\
$5.3(4.7,6.2)$ \\
$3.4(3.0,4.0)$ \\
$1.22(1.0,1.42)$ \\
$1.5(1.1,2.2)$ \\
$129(122,139)$ \\
$89(84,95)$ \\
50 \\
19 \\
12
\end{tabular}

${ }^{\mathrm{a} T w o-h o u r s . ~}$ 
$n=35$ participants ( $67 \%$ of total) met three criteria, $n=13$ (25\%) met four criteria and $n=4(8 \%)$ met five criteria; the distribution did not differ among groups (data not shown).

\section{Mixed-meal test}

Fasting values $>$ At day 14, PRED did not alter fasting glucose, while fasting C-peptide was diminished and glucagon was increased. Also, PRED decreased fasting insulin:glucose and insulin:glucagon ratios. The combination therapy of PRED+SITA merely resulted in increased fasting glucagon, while leaving the other fasting values undisturbed (Supplementary Table 1, see section on supplementary data given at the end of this article). DPP-4 activity was not altered by PRED monotherapy or PBO, but was significantly lowered in both PRED + SITA and SITA (data not shown).

Postprandial measures $>$ PRED vs $\mathrm{PBO}$ significantly increased postprandial $\mathrm{AUC}_{\text {glucose }}$ by $\sim 17 \%$, while PRED + SITA was not able to prevent this (Fig. 1 and Supplementary Table 2 , see section on supplementary data given at the end of this article). PRED vs PBO significantly lowered postprandial $\mathrm{AUC}_{\text {insulin }}$ but increased $\mathrm{AUC}_{\text {glucagon, }}$ resulting in decreased postprandial insulin:glucagon ratio. For PRED +SITA a similar reduction in $A U C_{\text {insulin }}$ or insulin:glucose ratio was observed. However, postprandial $\mathrm{AUC}_{\text {glucagon }}$ and insulin:glucagon ratio was not significantly different from PBO (Fig. 1 and Supplementary Table 2).

Using mathematical modeling to detail various aspects of $\beta$-cell function, we showed that PRED vs PBO significantly reduced glucose sensitivity by $\sim 53 \%$ and insulin secretion rate at fixed glucose reference of $5.5 \mathrm{mM}$ by $\sim 32 \%$. When combined, PRED + SITA comparably reduced glucose sensitivity $(P<0.001$ vs $\mathrm{PBO})$, while insulin secretion rate at fixed glucose reference of $5.5 \mathrm{mM}$ remained unaltered (nonsignificant vs $\mathrm{PBO}$ ). Other model-derived parameters, including total insulin secretion rate, rate sensitivity, and potentiation factor were not affected by either treatment (Table 2).

\section{Clamp procedure}

PRED vs PBO significantly reduced all parameters of $\beta$-cell function, as measured during the hyperglycemic part of the clamp, including 1st phase C-peptide secretion by $\sim 22 \%$, 2 nd phase by $\sim 30 \%$, and the response of C-peptide secretion to hyperglycemia-argininestimulation by $\sim 32 \%$. Concomitant SITA restored PRED- induced decrease in 1st and 2nd phase glucose-stimulated C-peptide secretion, but had no effect on the decline in arginine-stimulated C-peptide secretion (Fig. 2 and Supplementary Table 3, see section on supplementary data given at the end of this article). Hyperinsulinemiceuglycemic clamp-derived $M$-value was not altered by any treatment (Supplementary Table 3), nor was HOMA-IR (Supplementary Table 1).

\section{Cardiovascular risk factors}

At day 14, PRED or PRED + SITA vs PBO did not significantly alter body weight, systolic blood pressure, diastolic blood pressure, or fasting lipid profile, while it significantly increased HDL-cholesterol (Supplementary Table 4 , see section on supplementary data given at the end of this article).

At day 28, there were no differences in fasting or $2 \mathrm{~h}$ OGTT-values among the groups, indicating that all PREDinduced effects on glucose tolerance were back to baseline. Participants in both SITA and PRED+SITA (NB PREDtreatment halted after 14 days) were characterized by a lower, however not significant, systolic blood pressure at day 28 compared with PBO (Supplementary Table 4).

\section{Safety and tolerability}

Overall, compared with PBO no difference in frequency of reported adverse events during treatment with PRED and/or SITA was observed (data not shown). Frequently reported side effects for both drugs included upper respiratory tract infections, gastric discomfort/dyspepsia, and sleeplessness; these were generally mild and transient of nature.

\section{Conclusions}

In this study we explored, for the first time, the potential of concomitant DPP-4 inhibition to prevent diabetogenic side effects induced by GC treatment, particularly by protecting against GC-induced deterioration of pancreatic islet-cell function in high-risk individuals. The main findings were that, despite improvements in some aspects of islet-cell function, the DPP-4 inhibitor sitagliptin was not able to prevent GC-induced glucose intolerance, as assessed during a standardized meal-test in men with the metabolic syndrome.

We confirmed and extended previous findings, showing that sub-acute treatment with a high dose of prednisolone induced postprandial but not fasting 

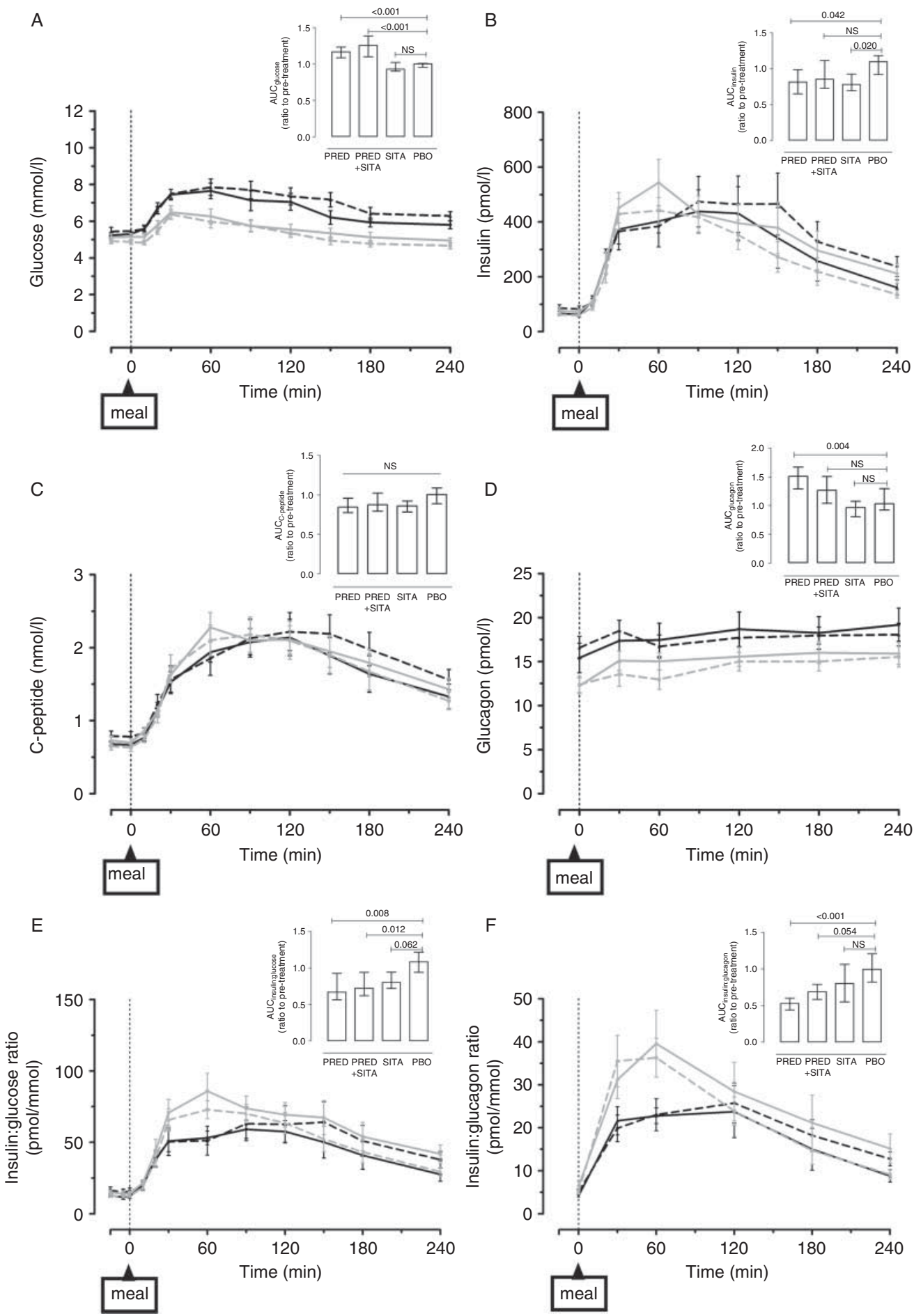

\section{Figure 1}

Results from the mixed-meal test. (A, B, C and D) Postprandial curves for glucose, insulin, C-peptide, and glucagon at day 14 ( $E$ and F) Ratio for the prevailing insulin to glucose levels (E) and insulin to glucagon levels (F). Bar graph insets represent the ratio to pre-treatment for the corresponding area under the curve (AUC). Values are median (interquartile range); $P$ values are Mann-Whitney $U$, in case of significant Kruskal-Wallis. Black solid line, prednisolone (PRED); black dashed line, prednisolone plus sitagliptin (PRED + SITA); gray dashed line, SITA; gray solid line, placebo (PBO). 


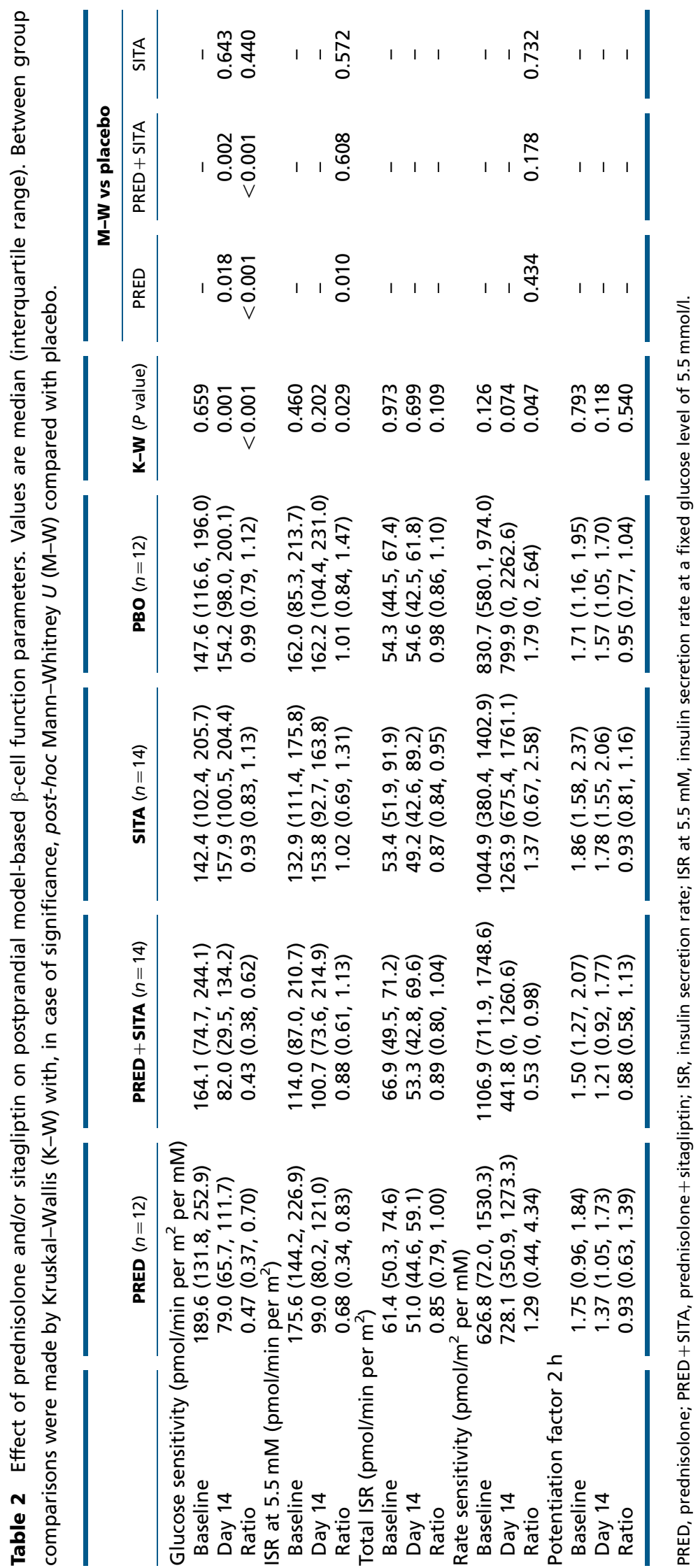



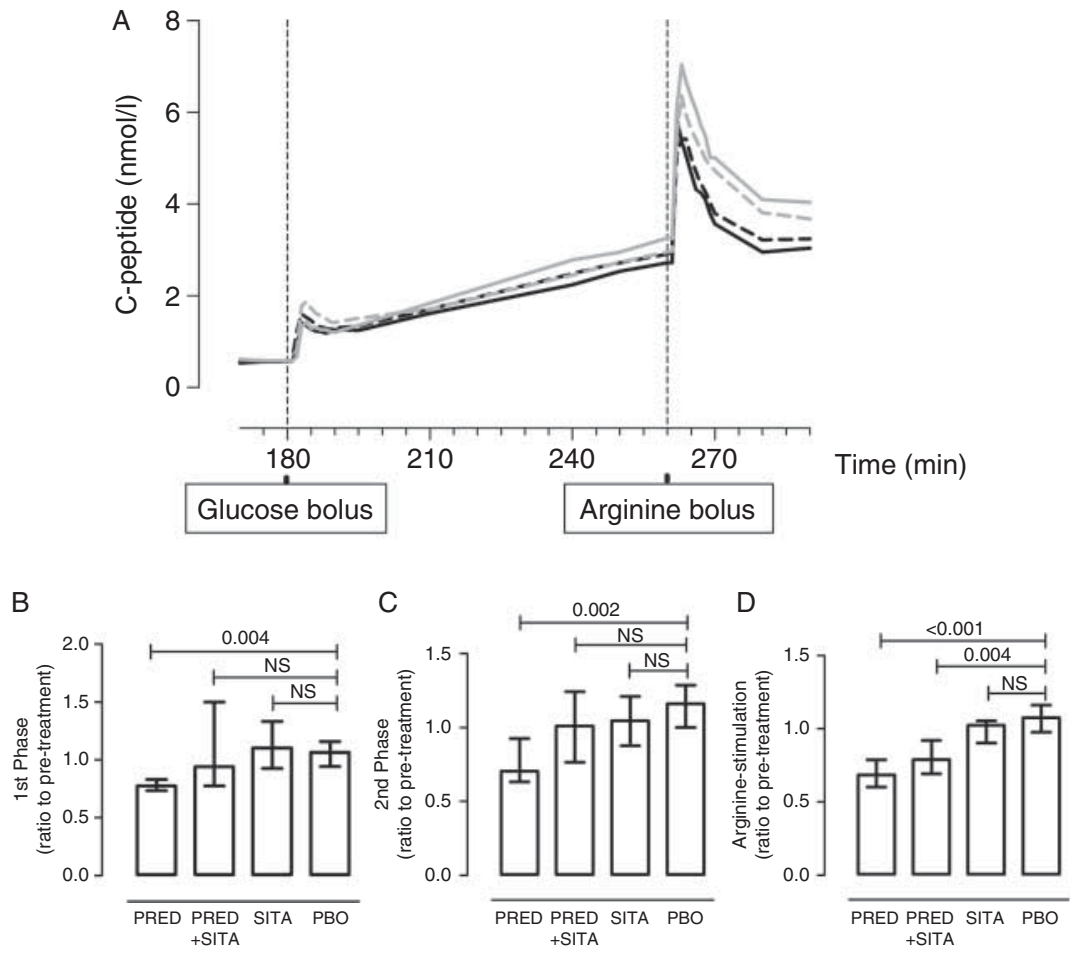

\section{Figure 2}

Results from the hyperglycemic clamp with argininestimulation. (A) C-peptide excursions on day 13 of treatment. ( $B, C$ and D) Ratio to pre-treatment for $\beta$-cell function parameters, quantified as area under the curve (AUC). (B) First phase (180-190 min) C-peptide secretion. (C) Second phase (190-260 min) C-peptide secretion. (D) Maximum C-peptide

hyperglycemia $(8,9)$ as well as islet-cell dysfunction $(5,6,15)$. The latter was characterized by impaired aspects of fasting, postprandial, and clamp-measured insulin secretion, in addition to increased fasting and mealrelated glucagon secretion. Impaired islet-cell function was previously demonstrated in rodent islet-cell cultures $(28,29)$, more recently in healthy young men $(5,6,15)$, and here also in men with the metabolic syndrome. Combined treatment of sitagliptin and prednisolone did not prevent meal-related glucose intolerance, as it failed to correct GC-induced disturbances of postprandial insulin response. Interestingly, sitagliptin administered with prednisolone improved some aspects of islet-cell function, particularly fasting C-peptide, clamp-measured 1st and 2nd phase C-peptide secretion and postprandial glucagon secretion. Also, a trend toward increased insulin secretion rate at fixed glucose level, as derived from dynamic $\beta$-cell function modeling, was noted. However, despite these improvements in GC-induced islet-cell dysfunction, secretion after arginine-stimulation (260-270 $\mathrm{min}$ ). Values are median (interquartile range). $P$ values are Mann-Whitney $U$, in case of significant Kruskal-Wallis. Black solid line, prednisolone (PRED); black dashed line, prednisolone plus sitagliptin (PRED + SITA); gray dashed line, SITA; gray solid line, placebo (PBO).

meal-related glucose intolerance was not prevented. A number of reasons could account for this seeming discrepancy. First, although the hyperglycemic clamp is generally considered the gold standard to measure pancreatic $\beta$-cell function, it represents a less physiological technique. Following ingestion of a nutrient load, several mechanisms in addition to plasma glucose levels, such as neurogenic stimuli, nonglucose nutrients, incretin hormones, or other gut-derived regulatory peptides contribute to insulin secretion. All these factors may potentially be altered by GCs and as such contribute to the persistent glucose intolerance despite improved clampmeasured $\beta$-cell function. Second, plasma glucose levels (15 $\mathrm{mM})$ during the hyperglycemic clamp were higher than the maximum glucose concentrations reached during the meal test $(\sim 8 \mathrm{mM})$. As incretin-enhanced insulin secretion from the $\beta$-cells is glucose dependent (30), the relative low postprandial glucose levels during the mixed-meal test in these nondiabetic individuals 
could not uncover the full potential of sitagliptin. Thirdly, GCs induce peripheral insulin resistance, resulting in increased hepatic glucose production, increased adipose tissue lipolysis, and impaired glucose disposal at the level of skeletal muscle (31). Although sitagliptin was shown by others to reduce hepatic glucose production in the postprandial state to some extent (32), by suppressing glucagon secretion, DPP-4 inhibitors, in general, fail to improve peripheral insulin sensitivity. These factors may explain why GC-induced glucose intolerance was not mitigated by sitagliptin, despite clamp-measured improvements in $\beta$-cell function.

At the level of the $\beta$ cell, several mechanisms have been suggested by which GCs impair insulin secretion, including GC-induced stress in endoplasmic reticulum (7), impairment of the efficacy of calcium on the secretory process, and reduction in protein kinase A (PKA)-mediated insulin release (28). Interestingly, incretins stimulate insulin secretion mainly through PKA activation (30). To our knowledge, no literature is currently available to describe the exact molecular mechanisms underlying GC-incretin interaction at the level of the $\alpha$ and $\beta$ cells. The fact that in humans the incretin effect is reduced by GCs, despite normal concentrations of (active) GLP1 (33, 34), suggests that GCs may impair GLP1-mediated activation of PKA. On the other hand, exenatide infusions, resulting in pharmacological levels of GLP1, were shown to prevent the acute diabetogenic effects of prednisolone in healthy volunteers (15). An additional mechanism by which GLP1 receptor agonist treatment, in contrast to DPP-4 inhibitors $(35,36)$, may prevent GC-induced hyperglycemia is by reducing gastric emptying rate - an effect attributed to the higher pharmacological levels of GLP1.

Two weeks of prednisolone treatment did not result in deterioration of a number of cardiovascular risk factors, including blood pressure, body weight, and body composition or lipid profiles. GC-induced hypertension has been shown previously to be dose dependent and occurs most often in elderly patients ( $>65$ years) (37) - our participants were middle aged - moreover, because $50 \%$ of the participants were already on anti-hypertensive treatment, a potential increase in blood pressure might have been masked. Furthermore, lipid profiles were not negatively altered. Possibly, the effects of GCs on fat accumulation in the liver, abdominal obesity, and plasma lipids require more prolonged exposure, such as is the case in patients with Cushing's disease (1).

Interestingly, we did not observe alterations in clampmeasured peripheral insulin sensitivity, generally considered a hallmark of GC-induced diabetogenic effects (4). However, previous studies were mostly done in healthy subjects with higher baseline insulin sensitivity ( $M$-value around $\sim 8 \mathrm{mg} / \mathrm{kg}$ per $\min )(6,15)$, whereas in this study participants were already suffering from severe insulin resistance at baseline ( $M$-value of $3.5 \mathrm{mg} / \mathrm{kg}$ per min). We chose to study the effects of GCs in the metabolic syndrome because patients with chronic inflammatory diseases, i.e. those requiring GC treatment, often have features of the metabolic syndrome $(16,38)$ independent of GC-use (38). In our study, short-term prednisolone exposure did not aggravate the already present severe insulin resistance. Similarly, in a previous study, chronic GC treatment in patients with rheumatoid arthritis did not further decrease insulin sensitivity compared with patients without GC-use (39).

Limitations of our study include its relative small size; however, previous studies of mechanism using similar methodology demonstrated meaningful effects in comparably sized protocols. Unfortunately, we were not able to include the exact number of participants for each group as calculated by the power-calculation. However, due to the strong significance of the differences observed, we do not expect that including a few more participants would have resulted in different outcomes. Besides, due to the inclusion and exclusion criteria, our study population was relatively homogenous (males, age-group, and metabolic syndrome criteria) allowing detection of small differences even in a relatively small number of participants. Other limitations include the absence of stable isotope use in the meal tests, which precludes conclusions on treatment-related changes in oral glucose uptake and hepatic glucose production, and the lack of postprandial plasma levels of incretin hormones. However, previous studies did not show an effect of GCs on (active) incretin levels $(6,33,34)$. We deliberately chose to include white men, as ethnicity and gender may be effect modifiers of several treatment-related study variables (40). However, this approach precludes generalization of the results. Finally, although metabolic syndrome is an appropriate model to study individuals at high risk to develop type 2 diabetes, this study population did not suffer from chronic systemic (high-grade) inflammation, which is typical for patients who are eligible for GC treatment. Therefore, the clinically relevant interrelationship among inflammation, GC treatment, and DPP-4 inhibition could not be assessed in this study.

In conclusion, the DPP-4 inhibitor sitagliptin did not prevent GC-induced glucose intolerance, despite improvements in various aspects of pancreatic islet-cell function in men with the metabolic syndrome, who are highly prone to develop type 2 diabetes. However, a longer term 
treatment or treatment combination in a clinically relevant population, i.e. patients that use GCs, may prove differently. Therefore, as steroid diabetes represents an important debilitating medical condition, future research should investigate whether incretin-based drugs or other islet-cell protecting agents can be harnessed as protective strategies against GC-induced postprandial hyperglycemia in a clinical setting.

\section{Supplementary data}

This is linked to the online version of the paper at http://dx.doi.org/10.1530/ EJE-13-0610.

\section{Declaration of interest}

R E van Genugten, D H van Raalte, M W Heymans, M H Muskiet, P J W Pouwels, M D M Ouwens, and A Mari declare no conflict of interest. M Diamant is consultant and speaker for Eli Lilly, MSD, Novo Nordisk, and Sanofi, consultant for Abbott, Astra-BMS, and Poxel Pharma. Through $M$ Diamant the VU University Medical Centre receives research grants from Amylin/Eli Lilly, MSD, Novo Nordisk, and Sanofi. M Diamant receives no personal payments in connection to the above-mentioned activities, but all payments are directly transferred to the Institutional Research Foundation. No shares and stocks.

\section{Funding}

R E van Genugten was supported by the EFSD/MSD clinical research program 2008 grant. In addition, for the reported study sitagliptin and matching placebo tablets were kindly provided by MSD BV, The Netherlands. MSD BV was not involved in the design of the study, data collection, data analysis, interpretation of results, and writing of the manuscript of this investigator-initiated trial. The opinions expressed in this manuscript are solely those of the authors and may not necessarily reflect those of MSD/Merck \& Co. M D M Ouwens is supported by the German Center for Diabetes Research (Deutsches Zentrum für Diabetesforschung, DZD).

\section{Author contribution statement}

R E van Genugten: performing of clinical experiments, data analysis, discussion of data, and writing manuscript. D H van Raalte: study design, discussion of data, and writing manuscript. M H Muskiet: data anlysis, discussion of data, and writing manuscript. M W Heymans: discussion of data and writing manuscript. P J W Pouwels: data analysis, discussion of data, and writing manuscript. M D M Ouwens: discussion of data and writing manuscript. A Mari: data analysis, discussion of data, and writing manuscript. M Diamant: study design, data analysis, discussion of data, and writing manuscript.

\section{Acknowledgements}

The authors thank J Boerop (Department of Internal Medicine, Diabetes Center, VU University Medical Center, Amsterdam, The Netherlands) for her excellent practical support. Furthermore, they thank their colleagues from the Department of Clinical Chemistry, J E Dijkstra and A C Heijboer (VU University Medical Center, Amsterdam, The Netherlands) for performing the laboratory measurements.

\section{References}

1 Schacke H, Docke WD \& Asadullah K. Mechanisms involved in the side effects of glucocorticoids. Pharmacology \& Therapeutics 200296 23-43. (doi:10.1016/S0163-7258(02)00297-8)

2 Clore JN \& Thurby-Hay L. Glucocorticoid-induced hyperglycemia. Endocrine Practice 200915 469-474. (doi:10.4158/EP08331.RAR)

3 Gulliford MC, Charlton J \& Latinovic R. Risk of diabetes associated with prescribed glucocorticoids in a large population. Diabetes Care 200629 2728-2729. (doi:10.2337/dc06-1499)

4 Van Raalte DH, Ouwens DM \& Diamant M. Novel insights into glucocorticoid-mediated diabetogenic effects: towards expansion of therapeutic options? European Journal of Clinical Investigation 200939 81-93. (doi:10.1111/j.1365-2362.2008.02067.x)

5 van Raalte DH, Nofrate V, Bunck MC, van Iersel T, Elassaiss Schaap J, Nässander UK, Heine RJ, Mari A, Dokter WH \& Diamant M. Acute and 2-week exposure to prednisolone impair different aspects of $\beta$-cell function in healthy men. European Journal of Endocrinology 2010162 729-735. (doi:10.1530/EJE-09-1034)

6 van Raalte DH, Kwa KA, van Genugten RE, Tushuizen ME, Holst JJ, Deacon CF, Karemaker JM, Heine RJ, Mari A \& Diamant M. Islet-cell dysfunction induced by glucocorticoid treatment: potential role for altered sympathovagal balance? Metabolism 201362 568-577. (doi:10.1016/j.metabol.2012.10.007)

7 Linssen MM, van Raalte DH, Toonen EJ, Alkema W, van der Zon GC, Dokter WH, Diamant M, Guigas B \& Ouwens DM. Prednisoloneinduced $\beta$ cell dysfunction is associated with impaired endoplasmic reticulum homeostasis in INS-1E cells. Cellular Signalling 201123 1708-1715. (doi:10.1016/j.cellsig.2011.06.002)

8 Burt MG, Willenberg VM, Petersons CJ, Smith MD, Ahern MJ \& Stranks SN. Screening for diabetes in patients with inflammatory rheumatological disease administered long-term prednisolone: a crosssectional study. Rheumatology 201251 1112-1119. (doi:10.1093/ rheumatology/kes003)

9 Yuen KC, McDaniel PA \& Riddle MC. Twenty-four-hour profiles of plasma glucose, insulin, C-peptide and free fatty acid in subjects with varying degrees of glucose tolerance following short-term, mediumdose prednisone $(20 \mathrm{mg} /$ day) treatment: evidence for differing effects on insulin secretion and action. Clinical Endocrinology 201277 224-232. (doi:10.1111/j.1365-2265.2011.04242.x)

10 Homik J, Cranney A, Shea B, Tugwell P, Wells G, Adachi R \& SuarezAlmazor M. Bisphosphonates for steroid induced osteoporosis. Cochrane Database of Systematic Reviews, 2000 CD001347. (doi:10.1002/ 14651858.CD001347)

11 Piper JM, Ray WA, Daugherty JR \& Griffin MR. Corticosteroid use and peptic ulcer disease: role of nonsteroidal anti-inflammatory drugs. Annals of Internal Medicine 1991114 735-740. (doi:10.7326/0003-4819114-9-735)

12 Drucker DJ \& Nauck MA. The incretin system: glucagon-like peptide-1 receptor agonists and dipeptidyl peptidase- 4 inhibitors in type 2 diabetes. Lancet 2006368 1696-1705. (doi:10.1016/S01406736(06)69705-5)

13 Baggio LL \& Drucker DJ. Therapeutic approaches to preserve islet mass in type 2 diabetes. Annual Review of Medicine 200657 265-281. (doi:10.1146/annurev.med.57.110104.115624)

14 Van Genugten RE, Van Raalte DH \& Diamant M. Dipeptidyl peptidase-4 inhibitors and preservation of pancreatic islet-cell function: a critical appraisal of the evidence. Diabetes, Obesity \& Metabolism 201214 101-111. (doi:10.1111/j.1463-1326.2011.01473.x)

15 Van Raalte DH, Van Genugten RE, Linssen MM, Ouwens DM \& Diamant M. Glucagon-like peptide-1 receptor agonist treatment prevents glucocorticoid-induced glucose intolerance and islet-cell dysfunction in humans. Diabetes Care 201134 412-417. (doi:10.2337/dc10-1677)

16 Bultink IE, Turkstra F, Diamant M, Dijkmans BA \& Voskuyl AE. Prevalence of and risk factors for the metabolic syndrome in women 
with systemic lupus erythematosus. Clinical and Experimental Rheumatology 200826 32-38.

17 Amori RE, Lau J \& Pittas AG. Efficacy and safety of incretin therapy in type 2 diabetes - systematic review and meta-analysis. Journal of the American Medical Association 2007298 194-206. (doi:10.1001/jama. 298.2.194)

18 Van Genugten RE, Moller-Goede DL, Van Raalte DH \& Diamant M. Extra-pancreatic effects of incretin-based therapies: potential benefit for cardiovascular risk management in type 2 diabetes. Diabetes, Obesity \& Metabolism 201315 593-606. (doi:10.1111/dom.12050)

19 Walker BR. Glucocorticoids and cardiovascular disease. European Journal of Endocrinology 2007157 545-559. (doi:10.1530/EJE-07-0455)

20 Alberti KG, Zimmet P \& Shaw J. Metabolic syndrome - a new worldwide definition. A Consensus Statement from the International Diabetes Federation. Diabetic Medicine 200623 469-480. (doi:10.1111/j. 1464-5491.2006.01858.x)

21 DeFronzo RA, Tobin JD \& Andres R. Glucose clamp technique - method for quantifying insulin-secretion and resistance. American Journal of Physiology 1979237 E214-E223.

22 van der Zijl NJ, Goossens GH, Moors CC, van Raalte DH, Muskiet MH, Pouwels PJ, Blaak EE \& Diamant M. Ectopic fat storage in the pancreas, liver, and abdominal fat depots: impact on $\beta$-cell function in individuals with impaired glucose metabolism. Journal of Clinical Endocrinology and Metabolism 201196 459-467. (doi:10.1210/jc.2010-1722)

23 Mari A, Schmitz O, Gastaldelli A, Oestergaard T, Nyholm B \& Ferrannini E. Meal and oral glucose tests for assessment of $\beta$-cell function: modeling analysis in normal subjects. American Journal of Physiology. Endocrinology and Metabolism 2002283 E1159-E1166. (doi:10.1152/ajpendo.00093.2002)

24 Mari A, Tura A, Gastaldelli A \& Ferrannini E. Assessing insulin secretion by modeling in multiple-meal tests - role of potentiation. Diabetes 2002 51 S221-S226. (doi:10.2337/diabetes.51.2007.S221)

25 Levy JC, Matthews DR \& Hermans MP. Correct homeostasis model assessment (HOMA) evaluation uses the computer program. Diabetes Care 199821 2191-2192. (doi:10.2337/diacare.21.12.2191)

26 Utzschneider KM, Tong J, Montgomery B, Udayasankar J, Gerchman F, Marcovina SM, Watson CE, Ligueros-Saylan MA, Foley JE, Holst JJ et al. The dipeptidyl peptidase-4 inhibitor vildagliptin improves $\beta$-cell function and insulin sensitivity in subjects with impaired fasting glucose. Diabetes Care 200831 108-113. (doi:10.2337/dc07-1441)

27 Rosenstock J, Foley JE, Rendell M, Landin-Olsson M, Holst JJ, Deacon CF, Rochotte E \& Baron MA. Effects of the dipeptidyl peptidase-IV inhibitor vildagliptin on incretin hormones, islet function, and postprandial glycemia in subjects with impaired glucose tolerance. Diabetes Care 200831 30-35. (doi:10.2337/dc07-1616)

28 Lambillotte C, Gilon P \& Henquin JC. Direct glucocorticoid inhibition of insulin secretion - an in vitro study of dexamethasone effects in mouse islets. Journal of Clinical Investigation 199799 414-423. (doi:10.1172/JCI119175)

29 Gremlich S, Roduit R \& Thorens B. Dexamethasone induces posttranslational degradation of GLUT2 and inhibition of insulin secretion in isolated pancreatic $\beta$ cells - comparison with the effects of fatty acids. Journal of Biological Chemistry 1997272 3216-3222. (doi:10.1074/jbc. 272.48.30261)
30 Leech CA, Dzhura I, Chepurny OG, Kang G, Schwede F, Genieser HG \& Holz GG. Molecular physiology of glucagon-like peptide-1 insulin secretagogue action in pancreatic $\beta$ cells. Progress in Biophysics and Molecular Biology 2011107 236-247. (doi:10.1016/j.pbiomolbio.2011. 07.005)

31 van Raalte DH, Brands M, van der Zijl NJ, Muskiet MH, Pouwels PJ, Ackermans MT, Sauerwein HP, Serlie MJ \& Diamant M. Low-dose glucocorticoid treatment affects multiple aspects of intermediary metabolism in healthy humans: a randomised controlled trial. Diabetologia 201154 2103-2112. (doi:10.1007/s00125-011-2174-9)

32 Muscelli E, Casolaro A, Gastaldelli A, Mari A, Seghieri G, Astiarraga B, Chen Y, Alba M, Holst J \& Ferrannini E. Mechanisms for the antihyperglycemic effect of sitagliptin in patients with type 2 diabetes. Journal of Clinical Endocrinology and Metabolism 201297 2818-2826. (doi:10.1210/jc.2012-1205)

33 Jensen DH, Aaboe K, Henriksen JE, Vølund A, Holst JJ, Madsbad S \& Krarup T. Steroid-induced insulin resistance and impaired glucose tolerance are both associated with a progressive decline of incretin effect in first-degree relatives of patients with type 2 diabetes. Diabetologia 201255 1406-1416. (doi:10.1007/s00125-012-2459-7)

34 Hansen KB, Vilsboll T, Bagger JI, Holst JJ \& Knop FK. Reduced glucose tolerance and insulin resistance induced by steroid treatment, relative physical inactivity, and high-calorie diet impairs the incretin effect in healthy subjects. Journal of Clinical Endocrinology and Metabolism 2010 95 3309-3317. (doi:10.1210/jc.2010-0119)

35 DeFronzo RA, Okerson T, Viswanathan P, Guan X, Holcombe JH \& MacConell L. Effects of exenatide versus sitagliptin on postprandial glucose, insulin and glucagon secretion, gastric emptying, and caloric intake: a randomized, cross-over study. Current Medical Research and Opinion 200824 2943-2952. (doi:10.1185/03007990802418851)

36 Vella A, Bock G, Giesler PD, Burton DB, Serra DB, Saylan ML, Dunning BE, Foley JE, Rizza RA \& Camilleri M. Effects of dipeptidyl peptidase-4 inhibition on gastrointestinal function, meal appearance, and glucose metabolism in type 2 diabetes. Diabetes 200756 1475-1480. (doi:10.2337/db07-0136)

37 Sato A, Funder JW, Okubo M, Kubota E \& Saruta T. Glucocorticoidinduced hypertension in the elderly. Relation to serum calcium and family history of essential hypertension. American Journal of Hypertension 19958 823-828. (doi:10.1016/0895-7061(95)00149-J)

38 Toms TE, Panoulas VF, Douglas KM, Griffiths HR \& Kitas GD. Lack of association between glucocorticoid use and presence of the metabolic syndrome in patients with rheumatoid arthritis: a cross-sectional study. Arthritis Research \& Therapy 200810 R145. (doi:10.1186/ar2578)

39 Hoes JN, van der Goes MC, van Raalte DH, van der Zijl NJ, den Uyl D, Lems WF, Lafeber FP, Jacobs JW, Welsing PM, Diamant M et al. Glucose tolerance, insulin sensitivity and $\beta$-cell function in patients with rheumatoid arthritis treated with or without low-to-medium dose glucocorticoids. Annals of the Rheumatic Diseases 201170 1887-1894. (doi:10.1136/ard.2011.151464)

40 Simonis-Bik AM, Eekhoff EM, de Moor MH, Kramer MH, Boomsma DI, Heine RJ, Dekker JM, Maassen JA, 't Hart LM, Diamant M et al. Genetic influences on the insulin response of the $\beta$ cell to different secretagogues. Diabetologia 200952 2570-2577. (doi:10.1007/s00125009-1532-3)

Received 25 July 2013

Revised version received 21 October 2013

Accepted 2 December 2013 